\title{
Automated vs manual delineations of regions of interest- a comparison in commercially available perfusion MRI software
}

\author{
Ivana Galinovic ${ }^{*}$, Ann-Christin Ostwaldt ${ }^{2}$, Carina Soemmer ${ }^{3}$, Helena Bros $^{1}$, Benjamin Hotter ${ }^{1}$, Peter Brunecker ${ }^{1}$ \\ and Jochen B Fiebach ${ }^{1}$
}

\begin{abstract}
Background: In perfusion magnetic resonance imaging a manual approach to delineation of regions of interest is, due to rater bias and time intensive operator input, clinically less favorable than an automated approach would be. The goal of our study was to compare the performances of these approaches.

Methods: Using Stroketool, PMA and Perfscape/Neuroscape perfusion maps of cerebral blood flow, mean transit time and Tmax were created for 145 patients with acute ischemic stroke. Volumes of hypoperfused tissue were calculated using both a manual and an automated protocol, and the results compared between methods.

Results: The median difference between the automatically and manually derived volumes was up to $210 \mathrm{ml}$ in Perfscape/Neuroscape, $123 \mathrm{ml}$ in PMA and $135 \mathrm{ml}$ in Stroketool. Correlation coefficients between perfusion volumes and radiological and clinical outcome were much lower for the automatic volumes than for the manually derived ones.

Conclusions: The agreement of the two methods was very poor, with the automated use producing falsely exaggerated volumes of hypoperfused tissue. Software improvements are necessary to enable highly automated protocols to credibly assess perfusion deficits.
\end{abstract}

Keywords: Magnetic resonance imaging, Perfusion MRI, Acute ischemic stroke

\section{Background}

The increased use of the perfusion imaging (PI) diffusion-weighted imaging (DWI) mismatch hypothesis [1] in studies of acute ischemic stroke (AIS) and clinical practice $[1,2]$ is raising demands from software packages developed for volumetric calculations of hypoperfusion. Typically these programs offer the possibility to delineate a region of interest (ROI) manually but also, to a varying extent, automatically. Manual approaches to delineation are biased and require time intensive operator input. Therefore a mostly automated procedure, if accurate, would be preferred in clinical practice. The goal of our study was comparing the automated approach to the manual approach while using a number of different software packages.

\footnotetext{
* Correspondence: ivana.galinovic@charite.de

${ }^{1}$ Center for Stroke Research Berlin, Charité-Universitätsmedizin Berlin, Campus Benjamin Franklin, Hindenburgdamm 30, 12200 Berlin, Germany Full list of author information is available at the end of the article
}

\section{Methods}

The study design and cohort characteristics have been previously reported [3]. Briefly, using Stroketool (Digital Image Solutions, Germany, http://www.digitalimagesolutions.de), PMA (v3.2.0.4, ASIST, Japan, http://asist.umin.jp/index-e. $\mathrm{htm}$ ) and Perfscape/Neuroscape (Olea Medical SAS, France, http://www.olea-medical.com) perfusion maps of cerebral blood flow (CBF), mean transit time (MTT) and Tmax were calculated for 145 patients imaged within 24 hours of AIS. The inclusion criteria were: clinically and radiologically confirmed AIS, hypoperfusion on the initial PI examination (assessed by the attending neuroradiologist) and availability of a follow-up MRI scan. For each parameter map, three thresholds were applied. The Tmax thresholds were 4, 6 and 8 seconds of delay [4] and the MTT thresholds were 5, 6 and 8 seconds. As no uniform CBF scale was available, the three CBF thresholds were different across software. Both MTT and CBF thresholds were chosen empirically using a

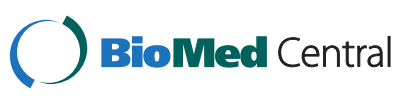


Table 1 Difference of ROI volumes between the automated and the manual protocol

\begin{tabular}{|c|c|c|c|c|c|}
\hline \multirow[t]{3}{*}{ Program } & Perfscape/Neuroscape & PMA & PMA with filtering * & Stroketool & Stroketool with filtering * \\
\hline & Median & Median & Median & Median & Median \\
\hline & IQR & IQR & IQR & IQR & IQR \\
\hline \multicolumn{6}{|l|}{ Parameter } \\
\hline \multirow[t]{2}{*}{ Tmax $4 \mathrm{~s}$} & 25.80 & 123.68 & 64.91 & 122.89 & 37.50 \\
\hline & $12.8-66.1$ & $75.2-206.8$ & $27.3-138.1$ & $75.6-176.5$ & $14.9-75.3$ \\
\hline \multirow[t]{2}{*}{$\operatorname{Tmax} 6 \mathrm{~s}$} & 12.74 & 29.48 & 4.83 & 53.18 & 7.75 \\
\hline & $4.1-34.4$ & $16.4-67.8$ & $1.0-22.4$ & $34.0-82.6$ & $1.8-18.3$ \\
\hline \multirow[t]{2}{*}{ Tmax $8 \mathrm{~s}$} & 8.05 & 23.16 & 2.68 & 31.78 & 1.67 \\
\hline & $2.6-28.1$ & $13.5-55.4$ & $0.4-15.8$ & $16.1-50.1$ & $0.2-6.5$ \\
\hline \multirow[t]{2}{*}{ MTT $5 \mathrm{~s}$} & 186.78 & 35.12 & 11.59 & 129.35 & 58.74 \\
\hline & $151.4-248.5$ & $17.8-87.8$ & $3.4-44.1$ & $82.5-225.7$ & $21.8-130.5$ \\
\hline MTT $6 \mathrm{~s}$ & $154.40114 .8-207.0$ & $16.768 .4-49.0$ & $4.340 .9-18.4$ & $80.1849 .1-143.6$ & $26.518 .0-63.7$ \\
\hline \multirow[t]{2}{*}{ MTT $8 \mathrm{~s}$} & 94.24 & 7.36 & 0.77 & 33.82 & 6.07 \\
\hline & $55.0-143.3$ & $3.8-17.4$ & $0.3-3.9$ & $20.9-57.6$ & $1.6-18.8$ \\
\hline \multirow[t]{2}{*}{ CBF highest threshold } & 209.92 & 54.35 & 23.05 & 134.50 & 67.28 \\
\hline & $160.0-262.8$ & $27.5-107.9$ & $7.6-64.4$ & 89.9-193.0 & $39.0-110.2$ \\
\hline \multirow[t]{2}{*}{ CBF medium threshold } & 151.93 & 36.14 & 10.51 & 87.28 & 35.27 \\
\hline & $97.6-212.2$ & $18.6-65.9$ & $3.7-36.5$ & $59.4-134.0$ & $18.1-61.7$ \\
\hline \multirow[t]{2}{*}{ CBF lowest threshold } & 63.66 & 23.40 & 4.33 & 53.95 & 12.73 \\
\hline & $39.6-119.4$ & $13.3-39.3$ & $1.4-15.3$ & $37.2-83.0$ & $6.6-28.4$ \\
\hline
\end{tabular}

CBF indicates cerebral blood flow; MTT mean transit time; IQR interquartile range. All values are in $\mathrm{ml}$.

* Values indicate volumes after additional CSF filtering in SPM8.

random sample of acute stroke patients. ROI volumes were calculated using a manual and an automated protocol. In the automated protocol, once the thresholds have been applied, no further post-processing was done. Maps created in PMA and Stroketool also underwent a second post-processing step in SPM8 (Wellcome Trust Centre for Neuroimaging, UK) to cut away scalp and spaces filled with cerebrospinal fluid (CSF). This was not necessary for Perfscape/Neuroscape due to the program's implemented filtering. In the manual protocol a human rater excluded, from the thresholded maps, areas unlikely to reflect credible hypoperfusion.

Radiological outcome was defined as the final lesion volume on follow-up FLAIR images. Clinical outcome was defined as the National Institute of Health Stroke Scale (NIHSS) score at the time of hospital discharge. All statistics were done in PASW Statistics 18. Analyses of correlations were performed using the Spearman signed-ranks correlation test.

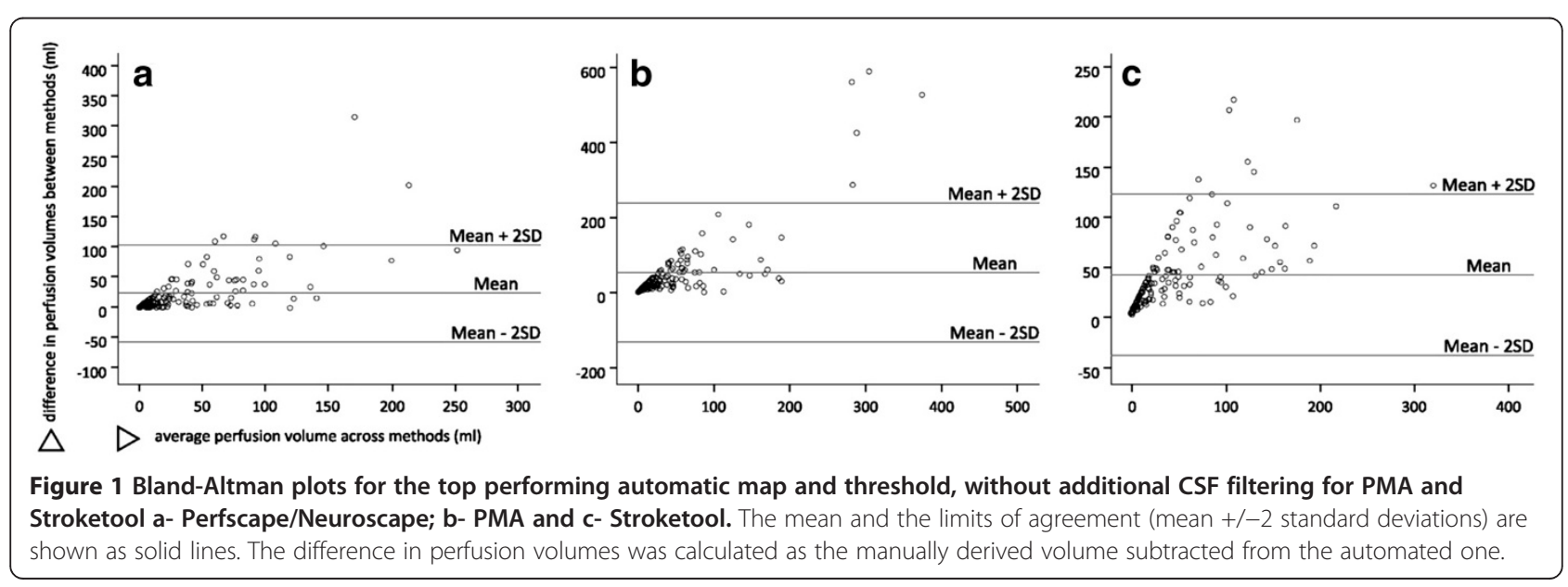




\section{Results}

The median final lesion volume was $6.55 \mathrm{ml}$ (IQR 0.8 $31.6 \mathrm{ml}$ ). The median difference between the automatically and manually derived volumes was up to $210 \mathrm{ml}$ (between 90\% and 386\%) in Perfscape/Neuroscape, $123 \mathrm{ml}$ (between 192\% and 1415\%) in PMA and $135 \mathrm{ml}$ (between $357 \%$ and $815 \%$ ) in Stroketool. (Table 1). Bland-Altman plots for agreement of methods are shown in Figure 1. All correlation coefficients between volumes of perfusion deficit and radiological and clinical outcome were considerably lower for the automatic volumes than for the manually derived ones. The top performing automated map was Tmax in all three programs. With additional filtering applied to automated volumes calculated in PMA and Stroketool the median difference between the automatically and manually derived volumes dropped down to a maximum of $64.91 \mathrm{ml}$ (between $44 \%$ and 238\%) and $67.28 \mathrm{ml}$ (between 33\% and 369\%), respectively (Table 1).

\section{Discussion}

The median volume of the perfusion deficit varied greatly based on the map and threshold and even across software for the same map and threshold. This discrepancy could in part be explained through the different choice of AIFs, the use of different deconvolution techniques, different implementations of the same calculation algorithm as well as differences in motion correction across different programs [5-7]. Our group had already conducted a study on a group of patients with no ischemia, using the same three software packages, and observed the presence of numerous artifacts; typically the cortex proximal to the skull and infratentorial cerebral and cerebellar tissue [8]. Programs without implemented CSF filtering also suffered from arti facts such as ventricles, eyeballs and scalp [8]. As expected, the same artifacts were present in our current patient cohort. This explains the weaker correlation coefficients and overshoot of the automated delineations as compared to the manual ones. Although most of the values on the Bland-Altman difference plot fall within the limits of agreement (Figure 1), these are much too broad with regards to the cohort's median lesion volumes and the fact that, based on location, even a lesion of a few $\mathrm{ml}$ can be clinically significant. Additional CSF filtering greatly reduced the differences between the automatic and the manual volumes, pointing to a need for implemented filtering.

\section{Conclusion}

In conclusion, current automated use of the here evaluated programs would lead to falsely exaggerated volumes of hypoperfused tissue in patients with AIS. However a number of improvements, such as algorithms for judging perfusion asymmetry between hemispheres and allowing selection of the vessel territory of expected hypoperfusion, could aid automated protocols in credibly assessing perfusion deficits.

\section{Competing interests}

The authors have no conflicts of interest to declare. All vendors provided software free of charge, no author received grants or fees from any of the involved software right owners and companies.

\section{Authors' contributions}

IG gathered the patient cohort, partly analyzed the imaging data, performed statistical analysis and drafted the manuscript. ACO participated in the analysis of the imaging data. CS participated in the analysis of the imaging data. HB participated in the analysis of the imaging data. $\mathrm{BH}$ participated in the gathering of the patient cohort and the drafting of the manuscript. PB performed the automated segment of data analysis. JBF was responsible for supervision and co-drafted the final version of the manuscript with IG. All authors read and approved the final manuscript.

\section{Acknowledgements}

No acknowledgements.

\section{Sources of funding}

The research leading to these results has received funding from the Federal Ministry of Education and Research via the grant Center for Stroke Research Berlin (01 EO 0801).

\section{Author details}

${ }^{1}$ Center for Stroke Research Berlin, Charité-Universitätsmedizin Berlin, Campus Benjamin Franklin, Hindenburgdamm 30, 12200 Berlin, Germany.

${ }^{2}$ International Graduate Program Medical Neurosciences,

Charite-Universitätsmedizin, Berlin, Luisenstrasse 56, 10117 Berlin, Germany.

${ }^{3}$ Charité-Universitätsmedizin, Berlin, Charitéplatz 1, 10117 Berlin, Germany.

Received: 24 August 2011 Accepted: 21 May 2012

Published: 18 July 2012

\section{References}

1. Hacke W, Albers G, Al-Rawi Y, Bogousslavsky J, Davalos A, Eliasziw M, et al: The Desmoteplase in Acute Ischemic Stroke Trial (DIAS): a phase II MRIbased 9-hour window acute stroke thrombolysis trial with intravenous desmoteplase. Stroke 2005, 36:66-73.

2. Kohrmann M, Juttler E, Fiebach JB, Huttner HB, Siebert S, Schwark C, et al: $M R I$ versus CT-based thrombolysis treatment within and beyond the $3 \mathrm{~h}$ time window after stroke onset: a cohort study. Lancet Neurol 2006, 5:661-667.

3. Galinovic I, Ostwaldt AC, Soemmer C, Bros H, Hotter B, Brunecker P, et al: Search for a map and threshold in perfusion MRI to accurately predict tissue fate: a protocol for assessing lesion growth in patients with a persistent vessel occlusion. Cerebrovasc Dis 2011, 32:186-193.

4. Olivot JM, Mlynash M, Thijs VN, Kemp S, Lansberg MG, Wechsler L, et al: Optimal Tmax threshold for predicting penumbral tissue in acute stroke. Stroke 2009, 40:469-475.

5. Kosior RK, Kosior JC, Frayne R: Improved dynamic susceptibility contrast (DSC)-MR perfusion estimates by motion correction. J Magn Reson Imaging 2007, 26:1167-72.

6. Kim J, Leirab EC, Callison RC, Ludwiga B, Moritani T, Magnotta VA, Madsen MT: Toward fully automated processing of dynamic susceptibility contrast perfusion MRI for acute ischemic cerebral stroke. Comput Methods Programs Biomed 2010, 98:204-213.

7. Yamada K, Wu O, Gonzalez RG, Bakker D, Copen WA, Weisskoff RM, Rosen BR, Yagi K, Nishimura T, Sorensen AG: Magnetic resonance perfusion-weighted imaging of acute cerebral infarction: effect of the calculation methods and underlying vasculopathy. Stroke 2002, 33(1):87-94.

8. Galinovic I, Brunecker P, Ostwaldt AC, Soemmer C, Hotter B, Fiebach JB: Fully automated postprocessing carries a risk of substantial overestimation of perfusion deficits in acute stroke magnetic resonance imaging. Cerebrovasc Dis 2011, 31:408-413.

doi:10.1186/1471-2342-12-16

Cite this article as: Galinovic et al: Automated vs manual delineations of regions of interest- a comparison in commercially available perfusion MRI software. BMC Medical Imaging 2012 12:16. 